30

\title{
Inactivation of Murine Norovirus and Fecal Coliforms by Ferrate(VI) in Secondary Effluent Wastewater
}

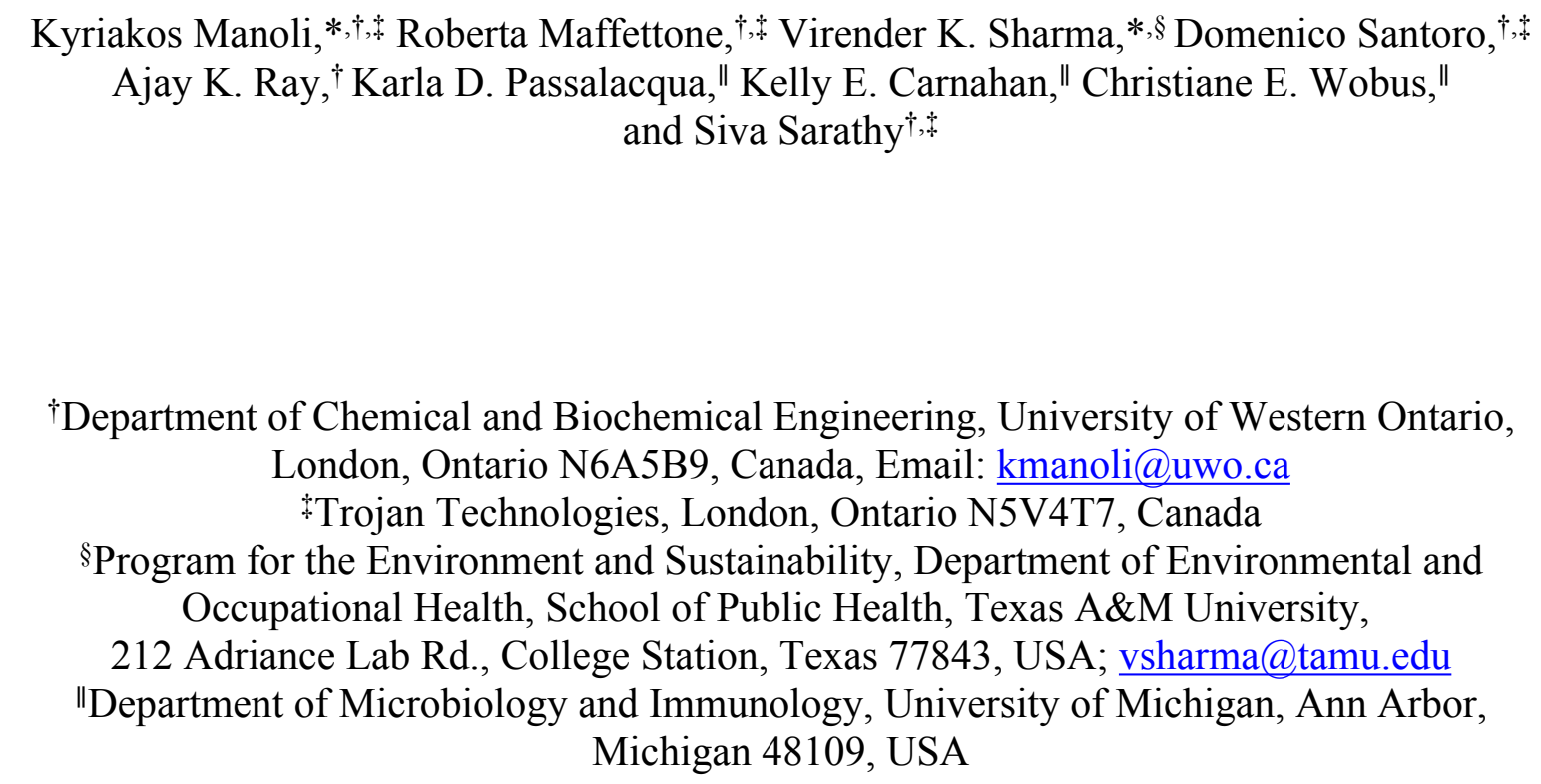


32 Chemicals. Phosphate buffer $(\mathrm{PB})$ solutions $(0.01 \mathrm{M})$ were prepared by diluting amounts of

$33 \mathrm{Na}_{2} \mathrm{HPO}_{4}$ and $\mathrm{NaH}_{2} \mathrm{PO}_{4} \cdot \mathrm{H}_{2} \mathrm{O}$ in DI water to achieve the desired $\mathrm{pH}$. The secondary effluent 34 wastewater (SEW) at $\mathrm{pH} 8.2$ was grab sampled (prior to disinfection step) at the Pottersburg 35 Wastewater Treatment Plant (London, Ontario, Canada), an activated sludge-based treatment 36 plant. Sodium phosphate dibasic $\left(\mathrm{Na}_{2} \mathrm{HPO}_{4} ; \geq 99 \%\right.$ purity) and sodium thiosulfate $\left(\mathrm{Na}_{2} \mathrm{~S}_{2} \mathrm{O}_{3}\right.$; $3799 \%$ purity) were purchased from Sigma-Aldrich, Canada. Sodium phosphate monobasic monohydrate $\left(\mathrm{NaH}_{2} \mathrm{PO}_{4} \cdot \mathrm{H}_{2} \mathrm{O} ; \geq 98 \%\right.$ purity) was purchased from Caledon Laboratory Chemicals 39 (Georgetown, Ontario, Canada). Polypropylene $(0.45 \mu \mathrm{m})$ and regenerated cellulose $(0.2 \mu \mathrm{m})$ syringe filters were acquired from VWR International (Mississauga, Ontario, Canada).

Fe(VI) Preparation and Quantification. The wet chemical method was used to synthesize solid 42 potassium ferrate(VI) $\left(\mathrm{K}_{2} \mathrm{FeO}_{4} ; 98 \%\right.$ purity). ${ }^{1} \mathrm{Fe}(\mathrm{VI})$ solutions were prepared prior to each experiment by diluting the desired amount (4-8 mg) of solid $\mathrm{K}_{2} \mathrm{Fe}^{\mathrm{VI}} \mathrm{O}_{4}$ to DI water (12-15 mL) followed by centrifugation at $3400 \mathrm{rpm}$ and $23 \pm 1{ }^{\circ} \mathrm{C}$. The concentration of $\mathrm{Fe}(\mathrm{VI})$ in the solution 45 was determined spectrophotometrically by measuring the absorbance at $510 \mathrm{~nm}$ using molar 46 absorptivity $\left(\varepsilon_{\mathrm{pH} 6.2}=476 \mathrm{M}^{-1} \mathrm{~cm}^{-1}, \varepsilon_{\mathrm{pH} 7.2}=663 \mathrm{M}^{-1} \mathrm{~cm}^{-1}, \varepsilon_{\mathrm{pH} 7.7}=908 \mathrm{M}^{-1} \mathrm{~cm}^{-1}, \varepsilon_{\mathrm{pH} .2}=1050\right.$

$47 \quad \mathrm{M}^{-1} \mathrm{~cm}^{-1}$ and $\left.\varepsilon_{\mathrm{pH} 9}=1150 \mathrm{M}^{-1} \mathrm{~cm}^{-1}\right) .^{2,3}$

48 Analytical Methods. The standard membrane filtration method (9222D) was applied to measure 49 the concentration (colony-forming units (CFU) $100 \mathrm{~mL}^{-1}$ ) of fecal coliforms (FC) in secondary 50 effluent wastewater $(\mathrm{SEW}) .^{4}$ Levels of chemical oxygen demand (dichromate method; 51 DOC316.53.01099) and nitrogen (s-TKN method; DOC316.53.01258) in SEW (Table S1) were 52 measured by performing the USEPA-approved Hach methods and testing kits (Hach Odyssey 
53 DR/2500; Hach, Loveland, Colorado, USA). Solids-related parameters (Table S1) of the SEW

54 were measured according to the standard methods. ${ }^{4}$ The UV transmittance (UVT) at $254 \mathrm{~nm}$ was

55 determined using a REALUVT meter (REALTECH, Whitby, Ontario, Canada), and the

56 absorbance at $400 \mathrm{~nm}$ was measured using the Hach spectrophotometer. Turbidity was measured

57 by a Hach 2100AN Turbidimeter (Table S1). Fisher Scientific accumet ${ }^{\mathrm{TM}}$ Portable pH (AP62)

58 and conductivity (AP65) Meters were used to measure the $\mathrm{pH}$ and conductivity, respectively.

\section{REFERENCES}

60 (1) Sharma, V. K.; Zboril, R.; Varma, R. S. Ferrates: greener oxidants with multimodal action 61 in water treatment technologies. Acc. Chem. Res. 2015, 48 (2), 182-191.

62 (2) Cho, M.; Lee, Y.; Choi, W.; Chung, H.; Yoon, J. Study on Fe(VI) species as a disinfectant: Quantitative evaluation and modeling for inactivating Escherichia coli. Water Res. 2006, 40 (19), 3580-3586.

65 (3) Luo, Z.; Strouse, M.; Jiang, J.-Q.; Sharma, V. K. Methodologies for the analytical determination of ferrate(VI): a review. J. Environ. Sci. Health. A. Tox. Hazard. Subst. Environ. Eng. 2011, 46 (5), 453-460.

68

(4) APHA. Standard methods for the examination of water and wastewater. In; American Public Health Association: Washington D.C., 1998. 

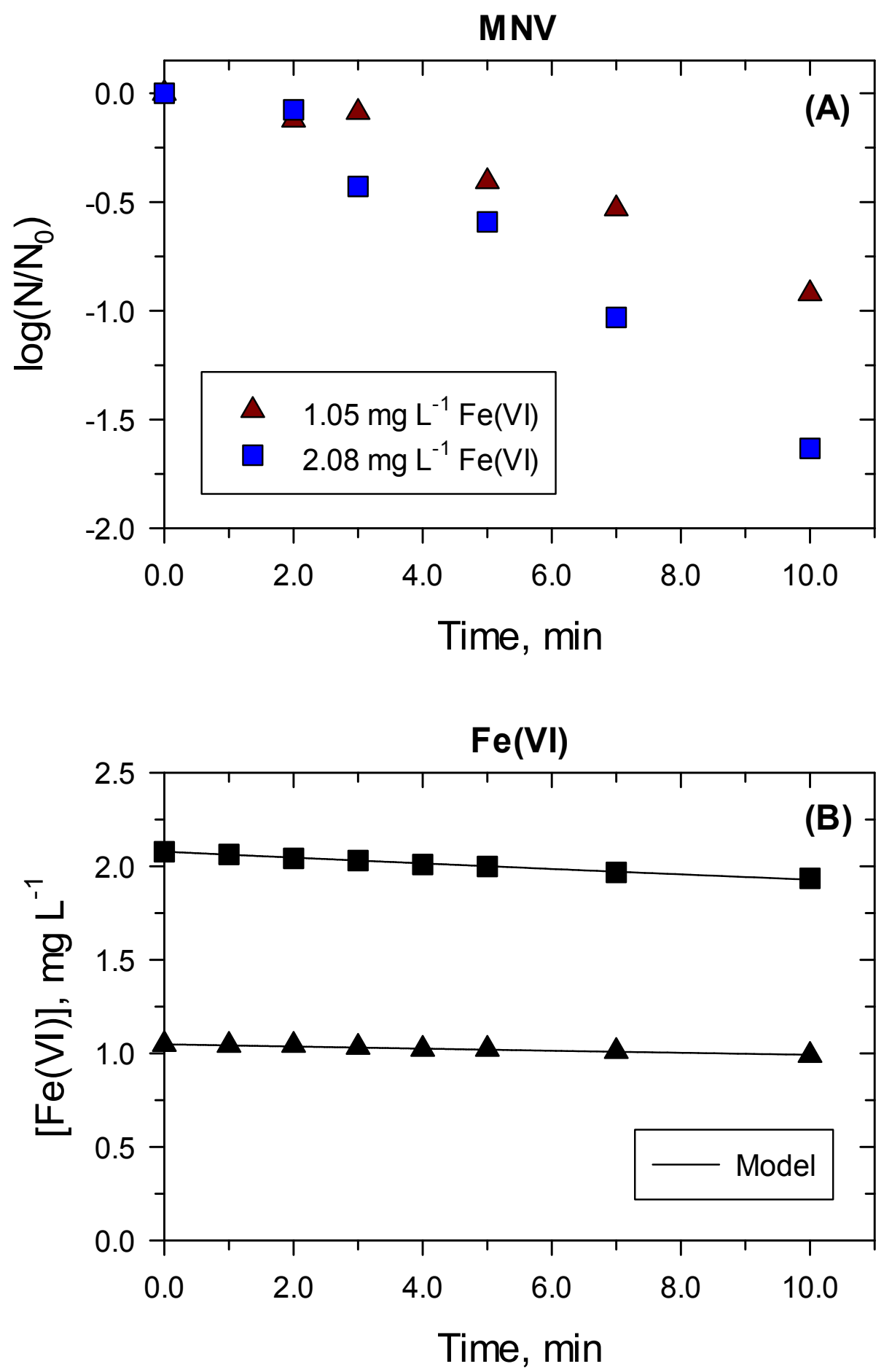

71

72 Figure S1. (A) Effect of Fe(VI) concentration on inactivation of MNV with time, and (B) Decay

73 of $\mathrm{Fe}(\mathrm{VI})$ with time at different concentrations of $\mathrm{Fe}(\mathrm{VI})$ (data fitted by eq 4). (Experimental

74 conditions: $[\mathrm{MNV}]_{1.05 \mathrm{mg} \mathrm{L}^{-1}}=917 \mathrm{PFU} \mathrm{mL}-1,[\mathrm{MNV}]_{2.08 \mathrm{mg} \mathrm{L}^{-1}}=429 \mathrm{PFU} \mathrm{mL}^{-1}, 0.01 \mathrm{M}$

75 phosphate buffer $\left.(\mathrm{pH}=8.2 \pm 0.1), \mathrm{T}=23 \pm 1^{\circ} \mathrm{C}\right)$. 


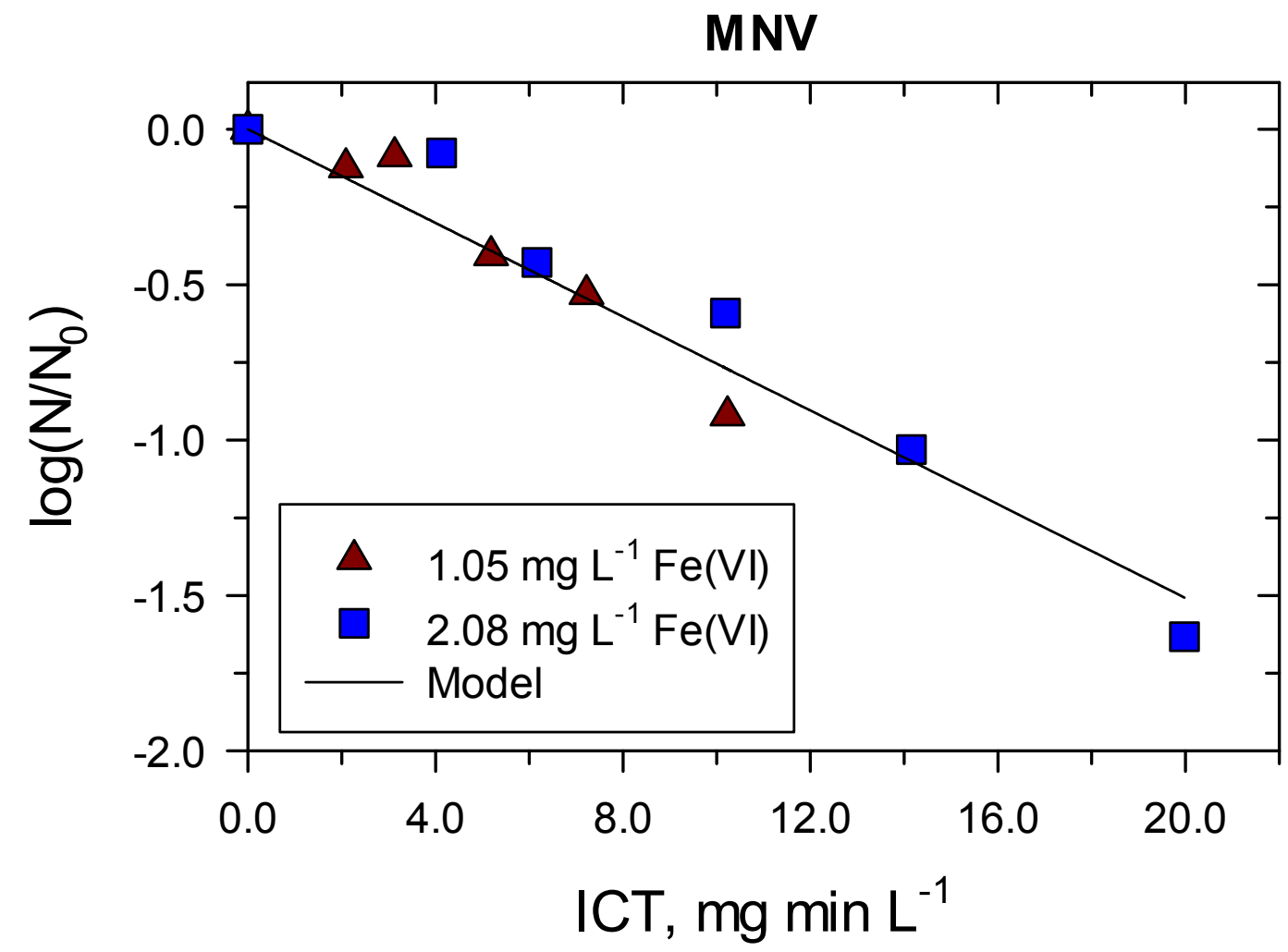

77 Figure S2. ICT dose response curve for the inactivation of MNV by $\mathrm{Fe}$ (VI) (Fitting data of 78 Figure S1(A) using Chick-Watson model (eq 1) (ICT calculated using eq 5). (Experimental 79 conditions: $[\mathrm{MNV}]_{1.05 \mathrm{mg} \mathrm{L}^{-1}=917 \mathrm{PFU} \mathrm{mL}}$, $[\mathrm{MNV}]_{2.08 \mathrm{mg} \mathrm{L}^{-1}=429 \mathrm{PFU} \mathrm{mL}} \mathrm{m}^{-1}, 0.01 \mathrm{M}$ 80 phosphate buffer $\left.(\mathrm{pH}=8.2 \pm 0.1), \mathrm{T}=23 \pm 1{ }^{\circ} \mathrm{C}\right)$.

81

82 

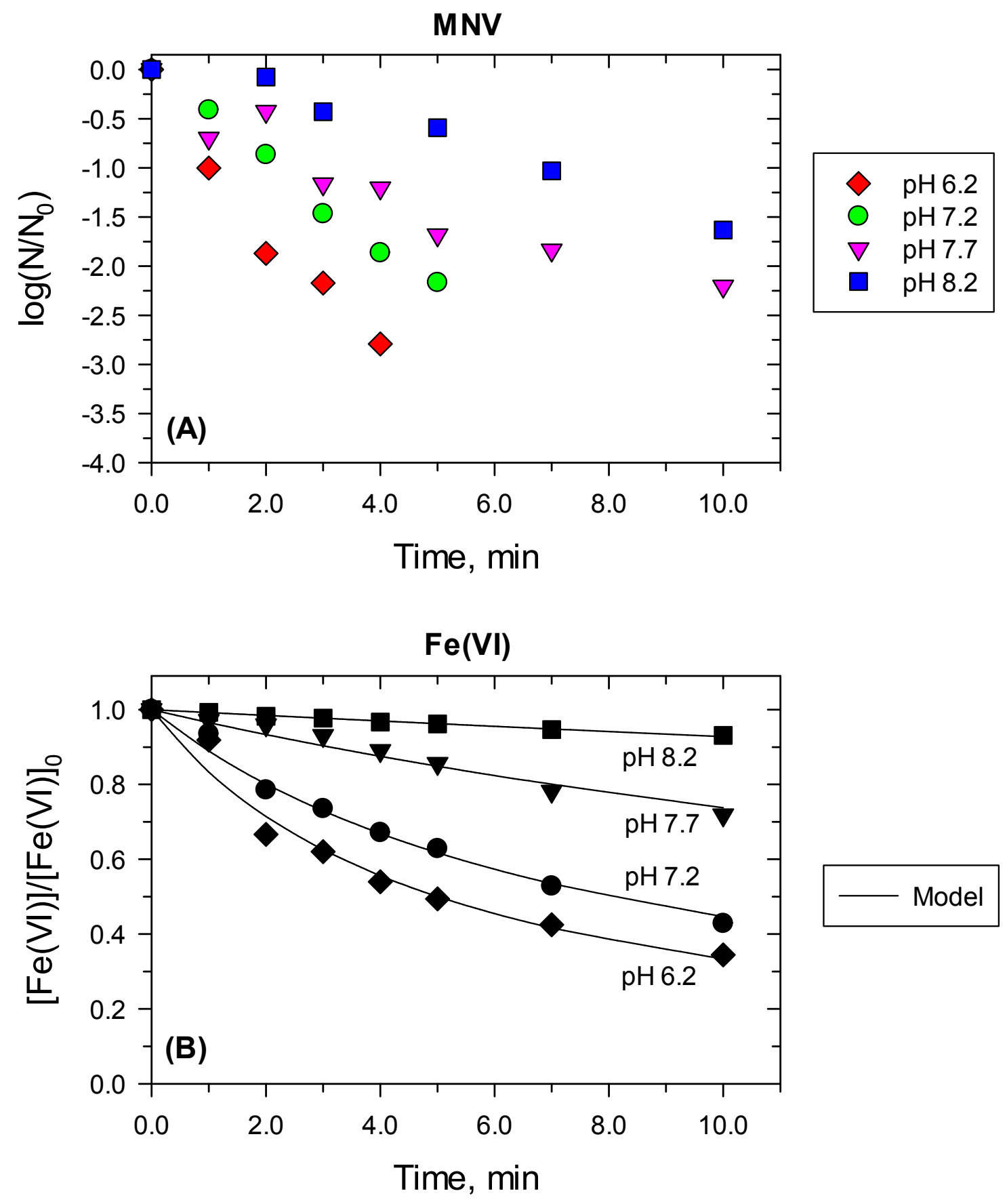

84 Figure S3. (A) Effect of $\mathrm{pH}$ on inactivation of MNV by Fe(VI) with time, and (B) Decay of $85 \mathrm{Fe}(\mathrm{VI})$ with time at different $\mathrm{pH}$ (data fitted by eq 4), in $0.01 \mathrm{M}$ phosphate buffer (PB).

86 (Experimental conditions: $[\mathrm{MNV}]_{\mathrm{pH}=6.2}=37126 \mathrm{PFU} \mathrm{mL}^{-1},[\mathrm{MNV}]_{\mathrm{pH}=7.2}=1467 \mathrm{PFU} \mathrm{mL}^{-1}$,

$87[\mathrm{MNV}]_{\mathrm{pH}=7.7}=4800 \mathrm{PFU} \mathrm{mL}^{-1},[\mathrm{MNV}]_{\mathrm{pH}=8.2}=429 \mathrm{PFU} \mathrm{mL}^{-1},[\mathrm{Fe}(\mathrm{VI})]_{\mathrm{pH}=6.2}=2.04 \mathrm{mg} \mathrm{L}^{-1}$,

$88[\mathrm{Fe}(\mathrm{VI})]_{\mathrm{pH}=7.2}=2.36 \mathrm{mg} \mathrm{L}^{-1},[\mathrm{Fe}(\mathrm{VI})]_{\mathrm{pH}=7.7}=2.14 \mathrm{mg} \mathrm{L}^{-1},[\mathrm{Fe}(\mathrm{VI})]_{\mathrm{pH}=8.2}=2.08 \mathrm{mg} \mathrm{L}^{-1}$, and $\mathrm{T}=$ $\left.8923 \pm 1^{\circ} \mathrm{C}\right)$. 


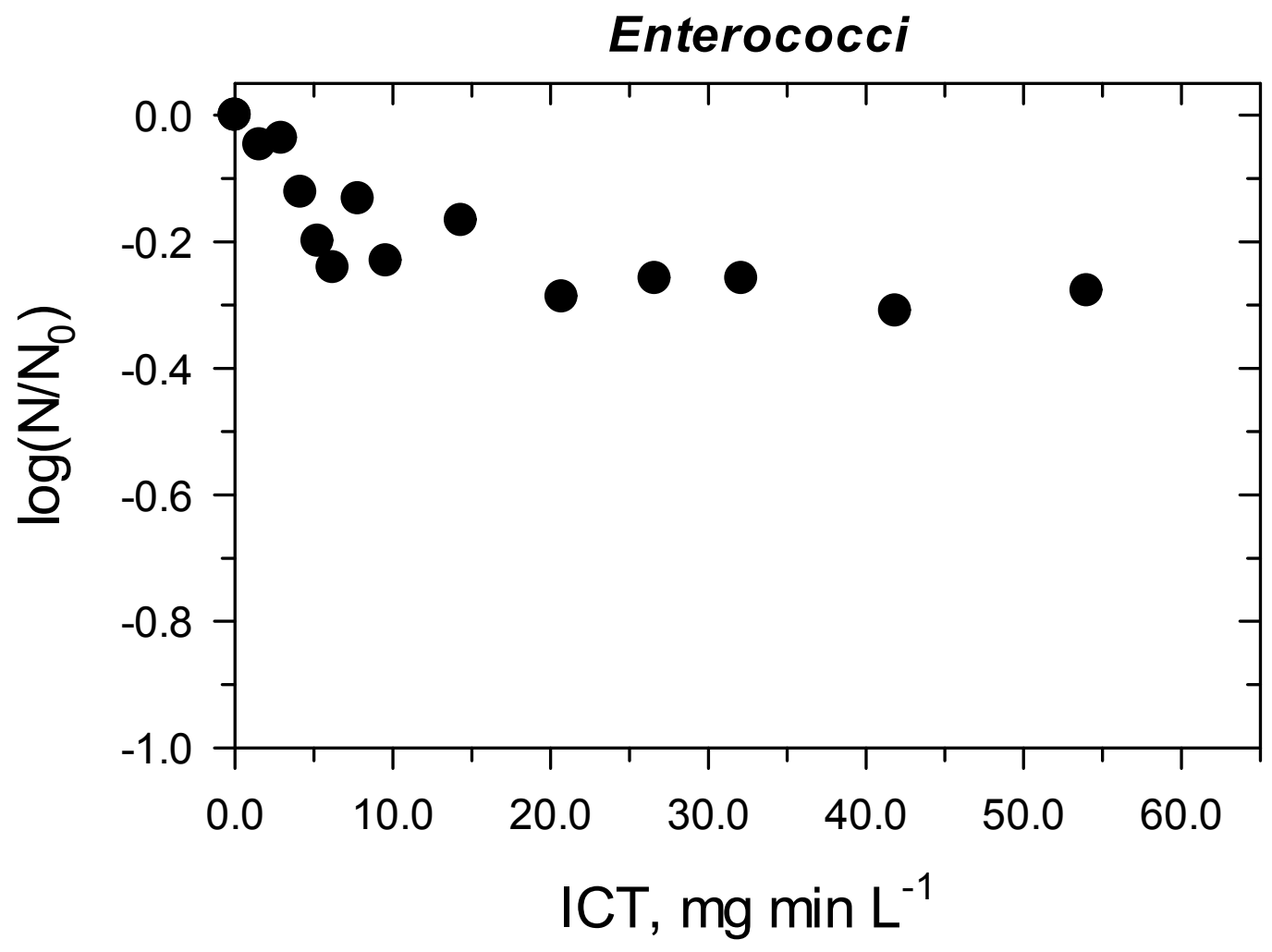

Figure S4. ICT dose response for the inactivation of enterococci by $\mathrm{Fe}(\mathrm{VI})$, in secondary 92 effluent wastewater (SEW). (Experimental conditions: [Enterococci] $=3798 \mathrm{CFU} 100 \mathrm{~mL}^{-1}$, $\left.93[\mathrm{Fe}(\mathrm{VI})]=1.16-8.29 \mathrm{mg} \mathrm{L}^{-1}, \mathrm{pH}=8.2 \pm 0.2, \mathrm{~T}=23 \pm 1{ }^{\circ} \mathrm{C}\right)$. 
97 Table S1. Water quality characteristics of the secondary effluent wastewater (SEW) used in the 98 study.

\begin{tabular}{|c|c|}
\hline Parameter & Value $^{a}$ \\
\hline Fecal coliforms, FC (CFU $\left.100 \mathrm{~mL}^{-1}\right)$ & $11440 \pm 303$ \\
\hline $\mathrm{pH}$ & $8.2 \pm 0.2$ \\
\hline Conductivity, $\left(\mu \mathrm{S} \mathrm{cm}^{-1}\right)$ & $1017 \pm 14$ \\
\hline $\mathrm{UV}_{400},\left(\mathrm{~cm}^{-1}\right)^{b}$ & $0.012 \pm 0.001$ \\
\hline $\mathrm{UVT}_{254},(\%)$ & $77.1 \pm 0.1$ \\
\hline $\mathrm{UV}_{254},\left(\mathrm{~cm}^{-1}\right)$ & $0.113 \pm 0.001$ \\
\hline Chemical Oxygen Demand, COD (mg L $\left.{ }^{-1}\right)$ & $37 \pm 3$ \\
\hline Soluble Chemical Oxygen Demand, SCOD $\left(\mathrm{mg} \mathrm{L}^{-1}\right)$ & $24 \pm 6$ \\
\hline Total Nitrogen, TN (mg N L-1) & $13.3 \pm 0.2$ \\
\hline Soluble Total Nitrogen, STN (mg N L $\left.{ }^{-1}\right)$ & $12.0 \pm 0.4$ \\
\hline Total Kjeldahl Nitrogen, TKN (mg N L-1) & $2.7 \pm 0.2$ \\
\hline Nitrites and Nitrates, $\mathrm{NO}_{2}^{-}-\mathrm{N}+\mathrm{NO}_{3}^{-}-\mathrm{N}\left(\mathrm{mg} \mathrm{N} \mathrm{L}^{-1}\right)$ & $10.6 \pm 0.1$ \\
\hline Particulate Organic Nitrogen, $\left(\mathrm{mg} \mathrm{N} \mathrm{L}^{-1}\right)$ & $1.3 \pm 0.4$ \\
\hline Ammonia + Soluble Organic Nitrogen $\left(\mathrm{mg} \mathrm{N} \mathrm{L}^{-1}\right)$ & $1.4 \pm 0.4$ \\
\hline Total Phosphorus, TP $\left(\mathrm{mg} \mathrm{P} \mathrm{L}^{-1}\right)^{c}$ & $0.45 \pm 0.13$ \\
\hline Turbidity, (NTU) & $5.0 \pm 0.1$ \\
\hline Total Solids, TS $\left(\mathrm{mg} \mathrm{L}^{-1}\right)$ & $653 \pm 10$ \\
\hline Total Suspended Solids, TSS (mg L-1) & $13 \pm 3$ \\
\hline Total Dissolved Solids, TDS (mg L $\left.{ }^{-1}\right)$ & $640 \pm 10$ \\
\hline
\end{tabular}

${ }^{a}$ Average \pm standard deviation of triplicate analysis of the grab sampled SEW

$100{ }^{b}$ Indication of color

101

${ }^{c}$ Measured by the wastewater treatment plant at the same day we collected the SEW

102 
105 Table S2. Kinetic parameters determined in this study for the inactivation of MNV by Fe(VI) $106\left(k_{\mathrm{d}}\right)$ and the decomposition of $\mathrm{Fe}(\mathrm{VI})\left(k_{2}\right)$ in $0.01 \mathrm{M}$ phosphate buffer.

\begin{tabular}{|c|c|c|c|c|}
\hline $\mathrm{pH}$ & $\begin{array}{c}{[\mathrm{MNV}]} \\
(\mathrm{PFU} \mathrm{mL}\end{array}$ & $\begin{array}{c}{[\mathrm{Fe}(\mathrm{VI})]} \\
\left(\mathrm{mg} \mathrm{L}^{-1}\right)\end{array}$ & $\begin{array}{c}k_{2}, 10^{-1} \\
\left(\mathrm{~L} \mathrm{mg}^{-1} \mathrm{~min}^{-1}\right)\end{array}$ & $\begin{array}{c}k_{\mathrm{d}}, 10^{-1} \\
\left(\mathrm{~L} \mathrm{mg}^{-1} \mathrm{~min}^{-1}\right)\end{array}$ \\
\hline 6.2 & 37126 & 2.04 & $0.98 \pm 0.06$ & $4.80 \pm 0.17$ \\
\hline 7.2 & 935 & 1.16 & $0.49 \pm 0.02$ & \multirow{2}{*}{$2.23 \pm 0.06$} \\
\hline 7.2 & 1467 & 2.36 & $0.53 \pm 0.02$ & $1.39 \pm 0.10$ \\
\hline 7.7 & 4800 & 2.14 & $0.17 \pm 0.01$ & \multirow{2}{*}{$0.75 \pm 0.04$} \\
\hline 8.2 & 917 & 1.05 & $0.05 \pm 0.002$ & \\
\hline 8.2 & 429 & 2.08 & $0.04 \pm 0.001$ & \\
\hline
\end{tabular}

107 
Table S3. Kinetic parameters determined in this study for inactivating MNV and FC by Fe(VI) in secondary effluent wastewater.

\begin{tabular}{|c|c|c|c|c|c|c|c|c|c|c|}
\hline \multirow[t]{2}{*}{$\mathrm{pH}$} & \multirow{2}{*}{$\begin{array}{c}{[\mathrm{MNV}]} \\
\left(\mathrm{PFU} \mathrm{mL}^{-1}\right)\end{array}$} & \multirow{2}{*}{$\begin{array}{l}{[\mathrm{Fe}(\mathrm{VI})]} \\
\left(\mathrm{mg} \mathrm{L}^{-1}\right)\end{array}$} & \multirow{2}{*}{$\begin{array}{c}{[\mathrm{FC}]} \\
(\mathrm{CFU} \\
\left.(100 \mathrm{~mL})^{-1}\right)\end{array}$} & \multirow{2}{*}{$\begin{array}{c}\mathrm{D} \\
\left(\mathrm{mg} \mathrm{L}^{-1}\right)\end{array}$} & \multirow{2}{*}{$\begin{array}{c}k_{1} \\
\left(\mathrm{~min}^{-1}\right)\end{array}$} & $\mathrm{MNV}^{\mathrm{a}}$ & \multicolumn{4}{|c|}{$\mathrm{FC}^{\mathrm{b}}$} \\
\hline & & & & & & $\begin{array}{c}k_{\mathrm{d}}, 10^{-1} \\
\left(\mathrm{~L} \mathrm{mg}^{-1} \min ^{-1}\right)\end{array}$ & $\beta$ & $\begin{array}{c}k_{\mathrm{d}} \\
\left(\mathrm{L}^{\mathrm{m}} \mathrm{mg}^{-\mathrm{m}} \min ^{-\mathrm{m}}\right)\end{array}$ & $\mathrm{m}$ & $\begin{array}{c}k_{\mathrm{p}} \\
\left(\mathrm{L} \mathrm{mg}^{-1} \min ^{-1}\right)\end{array}$ \\
\hline 8.2 & - & 1.16 & 11372 & $0.21 \pm 0.04$ & $0.37 \pm 0.04$ & - & \multirow{3}{*}{$0.017 \pm 0.003$} & \multirow{3}{*}{$2.08 \pm 0.05$} & \multirow{3}{*}{$1.08 \pm 0.06$} & \multirow{3}{*}{$0.03 \pm 0.018$} \\
\hline 8.2 & 32327 & 2.06 & 11771 & $0.41 \pm 0.09$ & $0.12 \pm 0.02$ & \multirow{2}{*}{1.3} & & & & \\
\hline 8.2 & 24123 & 8.29 & 11177 & $0.57 \pm 0.16$ & $0.08 \pm 0.004$ & & & & & \\
\hline
\end{tabular}


121 Table S4. Model predicted ICT values required for 1-, 2-, 3-, and 4- $\log _{10}$ reduction of murine 122 norovirus (MNV) and fecal coliforms (FC) by Fe(VI) in secondary effluent wastewater.

123

124

Reduction

ICT (mg min $\mathrm{L}^{-1}$ )

125

126

$127 \quad 1-\log _{10}$

$128 \quad 2-\log _{10}$

$129 \quad 3-\log _{10}$

$130 \quad 4-\log _{10}$

FC

MNV

131

132 